\title{
Residual Disease Following Conization of Women with Stage IA-IB1 Cervical Carcinoma in a High Incidence Region
}

\author{
Usanee Chatchotikawong, Irene Ruengkhachorn*, Chairat Leelaphatanadit
}

\begin{abstract}
Background: To determine rates of residual disease along with influencing factors in women with stage IA to IB1 cervical carcinoma after conization. Materials and Methods: A retrospective study was conducted of medical records of 198 stage IA to IB1 cervical carcinoma patients who had undergoing cervical conization followed by primary surgical treatment during 2006-2013. Independent factors correlating with residual carcinoma in subsequent surgical specimens were analyzed by stepwise regression analysis. Results: Mean age was 48.9 years. Cone specimens demonstrated free margins in 36 women $(18.8 \%)$. In case of having disease at margin, high-grade cervical intraepithelial neoplasia (CIN) and carcinoma were evidenced in 58 and 97 women, respectively. Pathology of subsequent specimens revealed residual carcinoma in 78 women $(39.4 \%)$, high-grade CIN or adenocarcinoma in situ (AIS) in $45(22.7 \%)$, and no residual pathology in $75(37.9 \%)$. Age more than 35 years, postmenopausal status, having symptoms, diseases or invasive lesions at conization margins or disease on endocervical aspect, and higher stage were significantly correlated with residual cancer in surgical treatment specimens. On regression analysis, postmenopause and stage were independent factors associated with residual carcinoma.Conclusions: Patient and tumor characteristics are predictive factors for residual cancer in the studied group of women.
\end{abstract}

Keywords: Cervix - cervical cancer - conization - residual disease - predicting factor

Asian Pac J Cancer Prev, 15 (17), 7383-7387

\section{Introduction}

Cervical carcinoma is the most common gynecological cancer in Thailand, and has age standardized rate of 16.7 in 100,000 population per year (Attasara and Sriplung, 2013). Early-stage cervical carcinoma is often asymptomatic and is increasingly detected by expansion of screening coverage. Conization is the standard method for diagnosis and staging of microscopic invasive lesions. The primary treatment is upon to the clinical staging by the International Federation of Gynecology and Obstetrics (FIGO) recommendations (Benedet et al., 2000; Pecorelli et al., 2009). Either primary radical surgery or concurrent chemo-radiation is accepted to have equal effectiveness for treatment of early-stage cervical cancer, which 70-90\% of five-year survival rate after treatment (Newton, 1975; Landoni et al., 1997).

The extension of surgical treatment in cervical cancer varies according to the risk of invasion and adjacent organs metastasis. Stage IA1 patients can be treated either by simple hysterectomy or as stage IA2, depending on the presence of lymph-vascular space invasion (LVSI). Surgical procedures for stage IA2 are modified radical hysterectomy and bilateral pelvic lymphadenectomy (MRHND) and for stage IB1 to IIA are radical hysterectomy and bilateral pelvic lymphadenectomy (RHND) (Benedet et al., 2000; Pecorelli et al., 2009; Shepherd, 2012). Previous studies reported rates of residual neoplasia in hysterectomy specimen of stages IA1 and IA 2 patients were $12 \%$ and $48 \%$, respectively (Suri et al., 2009; Lee et al., 2014). Adjuvant radiation therapy is necessary in cases of parametrium or surgical margin involvement, lymph node metastasis, large tumor size, deep invasion, or present of LVSI in order to decrease recurrence risk and increase survival (Peters et al., 2000; Rotman et al., 2006). The more extensive surgery and/or combination of adjuvant radiation treatment, the more complications occur, such as dysuria, constipation, and lymphedema.

For patient selection criteria to decrease extensive surgical treatment, the authors would like to determine $(i)$ the rate and type of residual diseases, and analyzed ( $i i)$ the influencing factors of residual cancer in women with stage IA to IB1 cervical carcinoma after diagnostic conization.

\section{Materials and Methods}

This study was a retrospective analysis of 198 women with stage IA and IB 1 cervical carcinoma with cervical conization followed by schedule for radical surgical treatment at Faculty of Medicine Siriraj Hospital, Mahidol 
University, Bangkok, Thailand, between January 1, 2006, and December 31, 2013. In our institute, conization and radical surgery were done by gynecological oncologists or resident/fellow under supervision. The purpose of diagnostic conization was to achieve entire lesions. Clinical staging was classified as in FIGO staging system. Radical surgery was scheduled within 6 weeks after clinical staging. The procedures for stage IA1 were simple hysterectomy with pelvic lymph node sampling in case of suspicious lymph node metastasis intra-operatively. The MRHND was utility for stage IA2, and RHND was for microscopic stage IB1 disease. Surgical procedures were stepped up to those for one higher stage when cone margin was positive for carcinoma. If frozen section confirmed parametrium or pelvic node metastasis, hysterectomy would be abandoned.

The cone tissue and hysterectomy specimens were prepared as standard guidelines; the section of cervix was evaluated at 12 o'clock and interval of 2-3 mm along endocervical canal. The margins of specimens were marked with adherent ink. Histopathology were classified as squamous cell carcinoma (SCCA), mucinous adenocarcinoma, adenosquamous carcinoma, mixed, and others including neuroendocrine carcinoma and undifferentiated carcinoma. SCCA were graded as well (grade 1), moderately (grade 2), and poorly differentiated (grade 3) by using a modified Broders' method. For other histological types, the grade was classified by the architectural complexity. The depth of stromal invasion (DSI) was the maximum of invasion measuring from basement membrane and categorized by the proportion of the cervical stromal invasion as inner third, middle third, and outer third. LVSI was defined as the presence of neoplastic cells within endothelium-lined spaces.

After approval by Siriraj Institutional Review Board, patients' characteristics, histopathology of conization, and subsequent surgical specimens were collected from the medical records of all women who met the studied criteria. The accumulated data were analyzed using SPSS version 18.0 (IBM,Armonk, NY, USA). Descriptive statistics were used for the baseline characteristics. Variables correlated with residual cancer in the subsequent surgical specimens and organ sites were analyzed by two-sided $\chi^{2}$ and Fisher exact tests as appropriate. Regression analysis was used for assessment of independent factors for predicting residual cancer in the subsequent surgical specimens. Variables with a P-value of less than 0.1 were included in a multivariable regression analysis to determine their association with residual cancer by estimated odds ratio (OR) and 95\% confidence interval (CI). A P-value of less than 0.05 was considered statistically significant.

\section{Results}

Total of 198 studied women, mean age was $48.85 \pm 9.64$ (range 25-78) years old and the mean body mass index was $24.59 \pm 4.57$ (range $16.41-43.28) \mathrm{kg} / \mathrm{m}^{2} .78$ women $(39.4 \%$ ) were menopause. 181 women $(91.4 \%)$ were multiparous with range of 1-9. The most common symptom was annual check-up in 152 women (76.8\%) and abnormal bleeding in 24 women (12.1\%). Pelvic examination of 15 women revealed abnormal cervical appearance which punchbiopsies confirmed cervical intraepithelial neoplasia (CIN). 183 women had abnormal cervical cytology preceding diagnostic conization, of which 11 women have no reports from primary hospital. 172 women with abnormal cytology had atypical squamous cells (ASC) in 15 women $(8.7 \%)$, low-grade squamous intraepithelial lesion (LSIL) in 2 women (1.2\%), high-grade squamous intraepithelial lesion (HSIL) in 95 women (55.2\%), SCCA in 35 women $(20.4 \%)$, atypical glandular cells (AGC) in 9 women $(5.2 \%)$, adenocarcinoma in situ (AIS) in 5 women $(2.9 \%)$, and adenocarcinoma in 11 women $(6.4 \%)$. In diagnostic conization, loop electrosurgical excision procedure (LEEP) in 169 women $(85.4 \%$ ) while cold-knife conization (CKC) in 29 women $(14.6 \%)$.

The most common histological results of conization specimens were SCCA in 139 women (70.2\%), the second most common was adenocarcinoma in 50 women (25.2\%). The cone-margins could not be evaluated in 7 women due to epithelial loss and/or thermal damage and/or disorientation caused by multiple pieces of cone specimens. 191 conization tissues were reported about margin status and found free of diseases in 36 women $(18.8 \%)$, positive ectocervical margin in 13 women $(6.8 \%)$, positive endocervical margin in 69 women $(36.1 \%)$, and positive both margins in 73 women (38.2\%). Of 155 positive cone margins, were CIN in 58 women (37.4\%) and carcinoma in 97 women (62.6\%). According to FIGO clinical staging, 88 women $(44.4 \%)$ were staged IA 1,33 women $(16.7 \%)$ IA 2 , and 77 women $(38.9 \%)$ IB 1 .

Simple hysterectomy with or without pelvic lymph

Table 1. Correlation of Pre-operative Factors and Extracervical Metastasis

\begin{tabular}{|c|c|c|c|c|}
\hline Factors & $\begin{array}{c}\text { Pelvic } \\
\text { nodes }(\%)\end{array}$ & $\begin{array}{l}\text { Parame- } \\
\text { trium(\%) }\end{array}$ & $\begin{array}{c}\text { Uterine } \\
\text { corpus }(\%)\end{array}$ & $\begin{array}{l}\text { Vagina } \\
(\%)\end{array}$ \\
\hline \multicolumn{5}{|l|}{ Cone histology } \\
\hline SCCA & 9/85 (10.6) & $9 / 80(11.3)$ & 4/136 (2.9) & $7 / 79(8.9)$ \\
\hline Non-SCCA & $0 / 52$ & $1 / 52$ (1.9) & $1 / 58 \quad(1.7)$ & $2 / 51(3.9)$ \\
\hline P-value & 0.013 & 0.088 & 1 & 0.481 \\
\hline \multicolumn{5}{|c|}{ Cone margin diseases } \\
\hline No carcinoma & $0 / 47$ & $2 / 43(4.7)$ & $3 / 93(3.2)$ & $1 / 43(2.3)$ \\
\hline Had carcinoma & $8 / 83(9.6)$ & $7 / 82(8.5)$ & $2 / 95(2.1)$ & $8 / 81(9.9)$ \\
\hline P-value & 0.05 & 0.717 & 0.681 & 0.161 \\
\hline \multicolumn{5}{|l|}{ FIGO stage } \\
\hline IA 1 & $0 / 27$ & $1 / 22(4.5)$ & $2 / 88(2.3)$ & $3 / 24(125)$ \\
\hline IA2 & $2 / 33(6.1)$ & $3 / 33(9.1)$ & $2 / 32(6.3)$ & $3 / 32(9.4)$ \\
\hline IB1 & $7 / 77(9.1)$ & 6/77 (7.8) & $1 / 74(1.4)$ & $3 / 74(4.1)$ \\
\hline P-value & 0.258 & 0.818 & 0.334 & 0.301 \\
\hline \multicolumn{2}{|c|}{ Pelvic node metastasis } & - & & \\
\hline \multicolumn{2}{|c|}{ No } & $5 / 122(4.1)$ & $4 / 126(3.2)$ & $7 / 121(5.8)$ \\
\hline \multicolumn{2}{|l|}{ Yes } & $5 / 9 \quad(55.6)$ & $1 / 7 \quad(14.3)$ & $1 / 7(14.3)$ \\
\hline \multicolumn{2}{|l|}{$\mathrm{P}$-value } & $<0.001$ & 0.24 & 0.371 \\
\hline \multicolumn{3}{|c|}{ Parametrium metastasis } & - & \\
\hline No & 4/121 (3.3) & & $3 / 122(2.5)$ & $4 / 122(3.3)$ \\
\hline Yes & $5 / 10(50.0)$ & & $2 / 6 \quad(33.3)$ & $3 / 6(50.0)$ \\
\hline P-value & $<0.001$ & & 0.017 & 0.002 \\
\hline \multicolumn{3}{|l|}{ Uterine metastasis } & - & \\
\hline No & $6 / 128(4.7)$ & $4 / 123(3.3)$ & & $8 / 125(6.4)$ \\
\hline Yes & $1 / 5 \quad(20.0)$ & $2 / 5 \quad(40.0)$ & & $1 / 5(20.0)$ \\
\hline P-value & 0.24 & 0.017 & & 0.306 \\
\hline \multicolumn{4}{|l|}{ Vaginal metastasis } & - \\
\hline No & $6 / 120(5.0)$ & $3 / 121(2.5)$ & 4/121 (3.3) & \\
\hline Yes & $1 / 8 \quad(12.5)$ & $3 / 7 \quad(42.9)$ & 1/9 (11.1) & \\
\hline P-value & 0.371 & 0.002 & 0.306 & \\
\hline
\end{tabular}

*Abbreviation: CIN, cervical intraepithelial neoplasia; FIGO, the International Federation of Gynecology and Obstetrics; SCCA, squamous cell carcinoma 
node sampling was performed in 66 women $(33.3 \%)$, MRHND in 12 women (6.1\%), RHND in 116 women $(58.6 \%)$, and abandoned hysterectomy in four women (2.0\%). Pelvic lymph node dissection or sampling was done in 137 women. Pathology of surgical specimens found no residual neoplasia in 75 women $(37.9 \%)$, CIN in 45 women $(22.7 \%$ ), and carcinoma in 78 women $(39.4 \%)$. Parametrium involvement was found in 10 women (7.6\%). Pelvic lymph node metastasis was found in 9 patients (6.6\%). In hysterectomy specimens of 194 women, found surgical margin involvement in 7 women (3.6\%); CIN in 4 and carcinoma in 3 women; LVSI in 21 women (10.8\%), and stromal invasion into middle third or deep third in 20 women each group (10.3\%). In women with stage IA1 and IA2, residual high-grade CIN was found in 29 (33.0\%) and $7(21.2 \%)$ women, whereas carcinoma was found in 18

Table 2. Correlated Factors with Residual Invasive Diseases in Subsequent Surgical Specimens

\begin{tabular}{|c|c|c|c|c|}
\hline Factors & $\begin{array}{c}\text { Total } \\
\mathrm{N}=198\end{array}$ & $\begin{array}{c}\text { Number of who } \\
\text { had residual } \\
\text { invasive }(\%)\end{array}$ & $\begin{array}{c}\text { Odds Ratio } \\
(95 \% \mathrm{CI})\end{array}$ & P-value \\
\hline \multicolumn{5}{|l|}{ Age (years) } \\
\hline$\leq 35$ & 15 & $1(6.7)$ & - & \\
\hline$>35$ & 183 & $77(42.1)$ & $\begin{array}{c}10.170 \\
(1.309-78.988)\end{array}$ & 0.007 \\
\hline \multicolumn{5}{|l|}{ Menopausal status } \\
\hline Premenopause & 120 & $39(32.5)$ & - & \\
\hline Postmenopause & 78 & $39(50.0)$ & $\begin{array}{c}2.077 \\
(1.156-3.730)\end{array}$ & 0.014 \\
\hline \multicolumn{5}{|l|}{ Presenting symptoms } \\
\hline Check-up & 152 & $54(35.5)$ & - & \\
\hline Had symptoms & 46 & $24(52.2)$ & $\begin{array}{c}1.980 \\
(1.016-3.858)\end{array}$ & 0.043 \\
\hline \multicolumn{5}{|l|}{ Cone margins ${ }^{\mathrm{a}}$} \\
\hline Free of disease & 36 & $7(19.4)$ & - & \\
\hline Positive for diseases & s 155 & $69(44.5)$ & $\begin{array}{c}3.324 \\
(1.373-8.046)\end{array}$ & 0.006 \\
\hline \multicolumn{5}{|l|}{ Cone margin diseases ${ }^{a}$} \\
\hline No carcinoma & 94 & $24(25.5)$ & - & \\
\hline Had carcinoma & 97 & $52(53.6)$ & $\begin{array}{c}3.370 \\
(1.828-6.213)\end{array}$ & $<0.001$ \\
\hline \multicolumn{5}{|l|}{ Cone margin aspects ${ }^{\mathrm{a}}$} \\
\hline Free/ positive Ecto. & 49 & $9(18.4)$ & - & \\
\hline Positive Endo. & 142 & $67(47.2)$ & $\begin{array}{c}3.970 \\
(1.794-8.789)\end{array}$ & $<0.001$ \\
\hline \multicolumn{5}{|l|}{ FIGO stage } \\
\hline IA 1 & 88 & $18(20.5)$ & - & \\
\hline IA2 & 33 & $15(45.5)$ & $\begin{array}{c}3.241 \\
(1.373-7.650)\end{array}$ & 0.006 \\
\hline IB1 & 77 & $45(58.4)$ & $\begin{array}{c}5.469 \\
(2.748-10.884)\end{array}$ & $<0.001$ \\
\hline
\end{tabular}

*Abbreviation: CI, confidence interval; Ecto., ectocervical margin; Endo., endocervical margin; FIGO, the International Federation of Gynecology and Obstetrics; ${ }^{a}, \mathrm{n}=191$

Table 3. Multivariate Analysis of Significant Factors for Predicting Residual Carcinoma

\begin{tabular}{llc}
\hline Factors & Odds Ratio (95\% CI) & P-value \\
\hline Age > 35 years & $6.938(0.726-66.309)$ & 0.093 \\
Postmenopause & $2.613(1.274-5.357)$ & 0.009 \\
Multiparity & $2.196(0.483-9.990)$ & 0.309 \\
Had symptoms & $2.101(0.913-4.837)$ & 0.081 \\
Positive cone margins & $1.255(0.178-8.866)$ & 0.82 \\
Positive Endo. & $1.931(0.363-10.259)$ & 0.44 \\
Cone margins had carcinoma & $1.437(0.617-3.345)$ & 0.4 \\
Stage IA2 & $2.889(1.087-7.678)$ & 0.033 \\
Stage IB1 & $5.733(2.446-13.436)$ & $<0.001$ \\
\hline *Abbreviation: CI, confidence interval; Endo., endocervical margin &
\end{tabular}

*Abbreviation: CI, confidence interval; Endo., endocervical margin
(20.5\%) and $15(45.5 \%)$ women. Microscopic IB1 disease had residual high-grade CIN and carcinoma in $9(11.7 \%)$ and $45(58.4 \%)$ women, respectively. 32/142 (22.5\%) and 67/142 (47.2\%) women with diseases at endocervical margin had residual high-grade CIN and carcinoma. For 97 women who had carcinoma at the cone margins, 18 women $(18.6 \%)$ had residual high-grade CIN, and 52 women $(53.6 \%)$ had residual carcinoma. Postoperative adjuvant concurrent chemo-irradiation was considered for 20 women $(10.1 \%)$ who had high-pathological risk factors for recurrence. There were no reports of adnexal metastasis in 138 women who were underwent bilateral salpingo-oophorectomy.

Table 1 showed the correlation of pre-operative factors and extracervical organs metastasis. The association of various factors with residual invasive diseases in surgical treatment specimens was showed in Table 2. Regression analysis of various factors to determine independent predictors for residual carcinoma was showed in Table 3.

\section{Discussion}

The rates of residual invasive diseases after diagnostic conization and extracervical involvement determine the extension of operative procedures for primary treatment of cervical carcinoma (Hefler et al., 2010; Lee et al., 2014). Furthermore, these can be used to select patients suitable for fertility preservative surgery. Lee et al., reported that the rate of residual CIN and cancer in 169 stage IA1 patients were $5.9 \%$ and $6.5 \%$, respectively (Lee et al., 2014). Hefler et al., showed that the rate of residual CIN and carcinoma in 102 stage IA1 SCCA patients were $34.3 \%$ and $7.8 \%$, respectively (Hefler et al., 2010). From the study of 42 patients with stage IA2, incidence of residual CIN or cancer was $48 \%$ (Suri et al., 2009). In this study, the rate of residual high-grade CIN and invasive lesions were $22.7 \%$ and $39.4 \%$, respectively. Unnecessary hysterectomy was found for $30 \%$ of stage IA 2 to IB 1 and $50 \%$ for stage IA 1 patients, because of no residual CIN or cancer in surgical treatment specimens. When compared stage by stage with previous studies, the current study had higher prevalence of residual CIN or cancer. This might be from higher rate of cervical neoplasia at conization margins, as a study of 129 patients with stage IA1 SCCA with positive cone margins that residual high-grade CIN and cancer were found in $44.2 \%$ and $15.5 \%$ of cases (Phongnarisorn et al., 2006).

The rates of adjacent organs metastasis such as pelvic lymph node or parametrium classified by stage were consistent with previous studies. In patients with stage IA1 cervical cancer had prevalence of pelvic lymph node metastasis of 0-2.9\% (Kodama et al., 2002; Poynor et al., 2006; Reynolds et al., 2010; Hou et al., 2011; Spoozak et al., 2012; Lee et al., 2014) and there were no parametrium metastasis (Poynor et al., 2006; Reynolds et al., 2010; Lee et al., 2014) except a study from Japan that reported the rate of $3.8 \%$ (Kodama et al., 2002). In stage IA2 patients had reported of pelvic lymph node metastasis of 0-3.5\% (Kodama et al., 2002; Poynor et al., 2006; Suri et al., 2009; Reynolds et al., 2010; Hou et al., 2011) and parametrium metastasis of $0-0.2 \%$ (Kodama 
Usanee Chatchotikawong et al

et al., 2002; Reynolds et al., 2010; Hou et al., 2011). The rate of parametrium metastasis in the present study was rather high, this supporting the benefit of parametrectomy in stage IA2 patients.

Furthermore, parametrium involvement was significantly correlated with the prevalence of pelvic lymph node, uterine or vaginal involvement and vice versa. The rate of no residual neoplasia and rate of adjacent organs metastasis might be useful for surgical treatment in stage IA1 to microscopic IB1 patients and physician had reluctance for extension of hysterectomy procedure, whether to include parametrectomy. For instance, pelvic lymphadenectomy was performed firstly with frozen pathology. If positive for carcinoma, parametrectomy can be operated right away. If not, parametrectomy is considered in selected case due to $4 \%$ of parametrium invasion. Up to now, there is no consensus of oncological outcomes between performing complete radical procedures or abortion of radical hysterectomy in cases of frozen section confirmed pelvic node metastasis. The authors think that morbidities of radical surgery in microscopic cervical cancer might be less than in macroscopic cervical cancer, so complete surgical procedures should be performed.

Older than 35 years, postmenopause, having clinical symptoms or diseases or cancer at conization margins or diseases at endocervical margin, and FIGO stage were significantly correlated with rate of residual cancer in subsequent surgical specimens. From previous study, advanced age more than 35 years was a significant predictor of residual high-grade CIN in stage IA1 SCCA patients as well (Hefler et al.,2010). This study encourages further surgery after conization in women older than 35 years old because of extremely high rate of residual carcinoma $(42.1 \%)$. In case fertility is still desired with age beyond this cut-off value, we should intensively counsel for this risk of residual malignancy and adverse pregnancy outcomes.

Anatomical change of cervix in postmenopausal women by inversion of T-zone into endocervical canal may result in invasive diseases invade higher than premenopause. Therefore, diagnostic conization was difficult to eradicate the whole lesions, so the rate of residual diseases was higher. The histopathology at margins of conization tissue either aspects of positive diseases and type of diseases were significant predictors for residual invasive disease. Based on the present data, in patients with diseases on endocervical cone margin or had carcinoma at any aspect of cone margins, the radical surgery would found residual high-grade CIN and invasive lesions were found around $20 \%$ and $50 \%$ after radical surgery, respectively, compared to $12.9-44.0 \%$ and 11.7$15.5 \%$, respectively in previous studies (Pongnarisorn et al., 2006; Lee et al., 2014). Roman et al., insisted on the significant correlation of internal margin with residual CIN and invasive lesion in stage IA1 SCCA patients $(49.0 \%$ vs $16.7 \%$ and $21.6 \%$ vs $3.3 \%$, respectively) (Roman et al., 1997).

This study found predominated of SCCA type in microscopic invasive cervical carcinoma. Likewise, the histological type of overall cervical cancer that found SCCA in $80-85 \%$, adenocarcinoma in $15 \%$ and adenosquamous cell carcinoma in 3-5\% (Pecorelli et al., 2009). As a result of diversity in depth or width estimation in adenocarcinoma of cervix, most gynecological oncologists disagree with stage IA cervical cancer classification. Therefore, RHND is the preferred treatment even in FIGO stage IA adenocarcinoma (Reynolds et al., 2010; Hou et al., 2011; Spoozak et al., 2012). Until now the United States and European researches have stated that early stage adenocarcinoma of cervix has metastasis, recurrence, and survival rates comparable to those of SCCA. Hence, surgical procedures in each stage of these two types of cervical cancer are similar (Covens et al., 1999; Poynor et al., 2006; Bisseling et al., 2007; Gien et al., 2010; Spoozak et al., 2012). These are consistent with the present study that the rate of residual diseases between SCCA and non-SCCA is not significantly different. Moreover, non-SCCA patients do not have significant higher rate of extracervical organs metastasis when compared with SCCA type.

FIGO stage IA2, IB1 and postmenopausal status were the independent predictors for residual invasive in studied population. These data support FIGO clinical staging system and the surgical procedures should be stepped higher according to increasing stage. Fortunately, postmenopausal patients are not in trouble clinically because there is no concern about fertility preserving surgery. The present data can be used for patient categorization to minimize the extension of surgical treatment in further study, for instance, integrating with human papilloma virus (HPV) testing or immunohistochemistry for prediction of residual carcinoma. The weakness of the current study was the limitation of retrospective study.

In conclusion, postmenopausal status and FIGO stage were independent risk factors of residual carcinoma after conization, in patients with stage IA to IB1 cervical carcinoma.

\section{Acknowledgements}

This research was supported by Siriraj Research Development Fund. The authors would like to appreciate Dr.Saowalak Hunnangkul, PhD., Epidemiology Unit, Siriraj Hospital for statistical analyses.

\section{References}

Attasara P, Sriplung H (2013). Cancer Incidence in Thailand. in: Khuhaprema T, Attasara P, Sriplung H, Wiangnon S, Sangrajrang S, eds. Cancer in Thailand Vol. VII. Bangkok: Lyon: International Agency for Research on Cancer, 8-76.

Benedet JL, Bender H, Jones H, et al (2000). FIGO staging classifications and clinical practice guidelines in the management of gynecologic cancers. FIGO Committee on Gynecologic Oncology. Int J Gynaecol Obstet, 70, 209-62.

Bisseling KC, Bekkers RL, Rome RM, Quinn MA (2007). Treatment of microinvasive adenocarcinoma of the uterine cervix: a retrospective study and review of the literature. Gynecol Oncol, 107, 424-30.

Covens A, Kirby J, Shaw P, Chapman W, Franseen E (1999). Prognostic factors for relapse and pelvic lymph node metastases in early stage I adenocarcinoma of the cervix. Gynecol Oncol, 74, 423-7. 
Gien LT, Beauchemin MC, Thomas G (2010). Adenocarcinoma: a unique cervical cancer. Gynecol Oncol, 116, 140-6.

Hefler LA, Polterauer S, Schneitter A, et al (2010). Repeat surgery in patients with cervical cancer stage FIGO IA1: a series of 156 cases and a review of the literature. Anticancer Res, 30, 565-8.

Hou J, Goldberg GL, Qualls CR, et al (2011). Risk factors for poor prognosis in microinvasive adenocarcinoma of the uterine cervix (IA1 and IA2): a pooled analysis. Gynecol Oncol, 121, 135-42.

Kodama J, Mizutani Y, Hongo A, et al (2002). Optimal surgery and diagnostic approach of stage IA2 squamous cell carcinoma of the cervix. Eur J Obstet Gynecol Reprod Biol, 101, 192-5.

Landoni F, Maneo A, Colombo A, et al (1997). Randomised study of radical surgery versus radiotherapy for stage Ib-IIa cervical cancer. Lancet, 350, 535-40.

Lee JY, Kim HS, Kim K, et al (2014). Safety of less aggressive surgery for stage IA1 squamous cell carcinoma of the cervix. J Obstet Gynaecol Res, 40, 1382-8.

Newton M (1975). Radical hysterectomy or radiotherapy for stage I cervical cancer. A prospective comparison with 5 and 10 years follow-up. Am J Obstet Gynecol, 123, 535-42.

Pecorelli S, Zigliani L, Odicino F (2009). Revised FIGO staging for carcinoma of the cervix. Int J Gynaecol Obstet, 105, 107-8.

Peters WA, 3rd, Liu PY, Barrett RJ, 2nd, et al (2000). Concurrent chemotherapy and pelvic radiation therapy compared with pelvic radiation therapy alone as adjuvant therapy after radical surgery in high-risk early-stage cancer of the cervix. J Clin Oncol, 18, 1606-13.

Phongnarisorn C, Srisomboon J, Khunamornpong S, et al (2006). The risk of residual neoplasia in women with microinvasive squamous cervical carcinoma and positive cone margins. Int J Gynecol Cancer, 16, 655-9.

Poynor EA, Marshall D, Sonoda Y, et al (2006). Clinicopathologic features of early adenocarcinoma of the cervix initially managed with cervical conization. Gynecol Oncol, 103, 960-5.

Shepherd JH (2012). Cervical cancer. Best Pract Res Clin Obstet Gynaecol, 26, 293-309.

Spoozak L, Lewin SN, Burke WM, et al (2012). Microinvasive adenocarcinoma of the cervix. Am J Obstet Gynecol, 206, 1-6.

Suri A, Frumovitz M, Milam MR, dos Reis R, Ramirez PT (2009). Preoperative pathologic findings associated with residual disease at radical hysterectomy in women with stage IA2 cervical cancer. Gynecol Oncol, 112, 110-3.

Reynolds EA, Tierney K, Keeney GL, et al (2010). Analysis of outcomes of microinvasive adenocarcinoma of the uterine cervix by treatment type. Obstet Gynecol, 116, 1150-7.

Roman LD, Felix JC, Muderspach LI, et al (1997). Risk of residual invasive disease in women with microinvasive squamous cancer in a conization specimen. Obstet Gynecol, 90, 759-64.

Rotman M, Sedlis A, Piedmonte MR, et al (2006). A phase III randomized trial of postoperative pelvic irradiation in Stage IB cervical carcinoma with poor prognostic features: followup of a gynecologic oncology group study. Int J Radiat Oncol Biol Phys, 65, 169-76. 